\title{
Simulation du comportement mécanique des alliages de titane pour les procédés de mise en forme à froid de produits plats
}

\author{
Benoit Revil-Baudard ${ }^{\mathrm{a}}$ et Elisabeth Massoni
}

Mines ParisTech, CEMEF - Centre de Mise en Forme des Matériaux, CNRS UMR 7635, BP 207, 1 rue Claude Daunesse, 06904 Sophia Antipolis Cedex, France

Reçu le 9 juillet 2010, accepté le 12 juillet 2010

\begin{abstract}
Résumé - Il est proposé, dans ce travail, un modèle de comportement mécanique anisotrope des alliages de titane de type $\alpha$ (structure hexagonale compacte). Le modèle élasto-plastique choisi se compose d'un écrouissage cinématique et isotrope et d'un critère de plasticité non quadratique anisotrope de type Cazacu et al. (Orthotropic yield criterion for hexagonal closed packed metals, Int. J. Plasticity 22 (2006) 11711194) adapté à ces matériaux à structure hexagonale compacte. L'implémentation de celui-ci dans un logiciel éléments-finis intègre deux repères de travail, dont celui des axes d'anisotropie réactualisé par le gradient de la transformation au cours de la mise en forme. Les paramètres des lois de comportement sont identifiés à partir d'essais de traction uniaxiale dans différentes directions. Des simulations, notamment de traction, de gonflage elliptique et d'emboutissage, ont permis de valider ce modèle. Une comparaison avec des cartographies de déformations, issues de mesure de champs, montre la bonne corrélation entre la simulation numérique et les tests expérimentaux pour les essais de gonflage elliptique.
\end{abstract}

Mots clés : Plasticité / anisotropie / écrouissage / simulation E.F. / alliages de titane / matériau hexagonal compact / formage à froid

\begin{abstract}
Simulation of titanium alloys behaviour for cold forming processes of metal sheets. In this paper, the mechanical behaviour of $\alpha$ titanium alloys is modelised for the cold forming processes. The elasto-plastic constitutive law is decomposed in an anisotropic plastic criterion, an isotropic hardening and a kinematic hardening. Non quadratic criteria have been developed by Cazacu et al. (Orthotropic yield criterion for hexagonal closed packed metals, Int. J. Plasticity 22 (2006) 1171-1194), to model the plasticity of hexagonal closed packed materials. The implementation of this model in a finite element software switches between two bases, the equilibrium is calculated in a reference basis and the anisotropy axes define a local basis, updated by the deformation gradient. An identification procedure, based on tensile tests, allows to define all the parameters needed to model the elasto-plastic behaviour. Simulation of cold forming processes (bulging and deep drawing) have been done to validate this model. Numerical results are compared with experimental data, obtained from speckles analysis.
\end{abstract}

Key words: Plasticity / anisotropy / constitutive laws / finite-element / titanium alloys / hexagonal closed packed material

\section{Introduction}

Les alliages de titane sont aujourd'hui fortement utilisés dans l'industrie aéronautique. Trois grandes classes d'alliage se démarquent selon leur microstructure : les alliages hexagonaux compacts de type $\alpha$, les alliages cubiques centrés de type $\beta$ et les alliages biphasiques $\alpha$ $\beta[2]$. L'objectif de ce travail est donc la modélisation du

\footnotetext{
a Auteur pour correspondance :

benoit.revil@mines-paristech.fr
}

comportement anisotrope d'alliage de titane $\alpha$ au cours de la mise en forme à froid. Hill propose un critère de plasticité simple et largement utilisé pour modéliser le comportement des aciers [3]. D'autres critères plus complexes et non quadratiques ont été développés pour la modélisation de l'anisotropie des alliages d'aluminium ou autres alliages métalliques [4]. Cazacu et al. [1] ont étendu cette famille de critères pour les matériaux hexagonaux compacts, tels les alliages de titane de type $\alpha$, afin de prendre en compte l'influence des phénomènes microstructuraux, tels que le glissement et le maclage 
spécifiques à cette structure cristalline. Le suivi des axes d'anisotropie au cours de la transformation permet une bonne modélisation des procédés de formage. D'après les conclusions de Duchêne et al. [5], une approche cinématique peut être utilisée pour la mise en place d'un modèle de comportement mécanique macroscopique. L'implémentation dans un logiciel de simulation par éléments-finis (E.F) implicite sera présentée pour simuler la mise en forme à froid de tôles d'alliage de titane. Afin de modéliser le comportement mécanique de celles-ci, des essais de traction uniaxiale seront exploités. Des comparaisons entre numérique et expérimental permettront de conclure sur l'efficacité de la modélisation choisie.

Dans cet article, les vecteurs sont représentés par $\{$. et les tenseurs par [.]. La transposée, l'inverse et la trace d'une matrice sont exprimées respectivement par [.] ${ }^{\mathrm{T}}$, $[.]^{-1}$ et $\operatorname{tr}($.$) , le produit tensoriel par \otimes$. La notation de Voigt, utilisée pour transformer un vecteur en tenseur, permet la simplification des écritures.

\section{Le comportement élasto-plastique anisotrope}

Le modèle élasto-plastique choisi nécessite la définition de plusieurs lois de comportement :

- un comportement élastique isotrope défini par la loi de Hooke : $\{\boldsymbol{\epsilon}\}=\frac{1+\nu}{E}\{\boldsymbol{S}\}$, où $E$ est le module de Young, $\nu$ le coefficient de Poisson, $\{\boldsymbol{\epsilon}\}$ et $\{\boldsymbol{S}\}$ les déviateurs des déformations et des contraintes. La matrice $[\boldsymbol{C}]$, carrée de taille 6 , est déduite à partir de la loi de Hooke telle que : $\{\boldsymbol{\epsilon}\}=[\boldsymbol{C}]\{\boldsymbol{S}\}$;

- une loi d'écrouissage cinématique pour définir le tenseur cinématique $\{\boldsymbol{X}\}$. La loi d'Armstrong-Frederick [6] permet une modélisation pour une large plage de déformations : $\{\dot{\boldsymbol{X}}\}=D\left\{\dot{\boldsymbol{\epsilon}}^{\mathrm{p}}\right\}-\xi\{\boldsymbol{X}\} \dot{\bar{\epsilon}}^{\mathrm{p}}, \operatorname{avec}\left\{\dot{\boldsymbol{\epsilon}}^{\mathrm{p}}\right\}$ le vecteur des taux de déformation et $\dot{\bar{\epsilon}}^{\mathrm{p}}$ le taux de déformation plastique équivalent. Cette loi associe un écrouissage cinématique linéaire, contrôlé par le paramètre du matériau $D$ et un terme modélisant la recristallisation dynamique contrôlée par le paramètre $\xi$;

- une surface convexe pour définir la surface de plasticité initiale : $f(\boldsymbol{S}-\boldsymbol{X})$;

- une loi d'écrouissage isotrope pour définir la contrainte d'écoulement $\sigma_{y}$.

Le comportement mécanique est donc défini par le critère de plasticité $F_{p}$ :

$$
F_{p}=f(\boldsymbol{S}-\boldsymbol{X})-\sigma_{y}, \quad F_{p} \leq 0 .
$$

Selon la valeur de $F_{p}$, le comportement du matériau est soit élastique $\left(F_{p}<0\right)$, soit élasto-plastique $\left(F_{p}=0\right)$. Nous nous intéresserons au comportement plastique, et plus particulièrement à l'influence du critère de plasticité. Deux critères ont été implémentés dans Forge ${ }^{\circledR}[7]$ : le critère de Hill 1948 [3] et le critère de Cazacu et al. 2006 [1]. On présente dans la suite les développements liés au critère de Cazacu.
Ce critère modélise un comportement non symétrique en traction et en compression. Ce critère est non quadratique et indépendant de la pression hydrostatique. Il s'exprime de la manière suivante :

$$
f(\tilde{\boldsymbol{S}})=\frac{1}{Q}\left(\sum_{i=1}^{3}\left(\left|\tilde{S}_{i}\right|-k \tilde{S}_{i}\right)^{a}\right)^{1 / a}
$$

où $\tilde{S}_{i}$ représente les valeurs principales du tenseur $[\tilde{\boldsymbol{S}}]$. Les deux paramètres $a$ et $k$ expriment le rapport entre les contraintes d'écoulement en traction et en compression. La condition de convexité de la surface de plasticité impose $a \geq 1$ et $-1 \leq k \leq 1$. Le tenseur $[\tilde{\boldsymbol{S}}]$ est calculé à partir de :

$$
\begin{aligned}
{\left[\begin{array}{l}
\tilde{S}_{11} \\
\tilde{S}_{22} \\
\tilde{S}_{33} \\
\tilde{S}_{12} \\
\tilde{S}_{23} \\
\tilde{S}_{13}
\end{array}\right]=} & {\left[\begin{array}{lllll}
L_{11} & L_{12} & L_{13} & & \\
L_{12} & L_{22} & L_{23} & & \\
L_{13} & L_{23} & L_{33} & & \\
& & L_{44} & \\
& & & L_{55} \\
& & & L_{66}
\end{array}\right] } \\
& \times\left[\begin{array}{l}
S_{11}-X_{11} \\
S_{22}-X_{22} \\
S_{33}-X_{33} \\
S_{12}-X_{12} \\
S_{23}-X_{23} \\
S_{13}-X_{13}
\end{array}\right]
\end{aligned}
$$

$L_{i j}$ sont 9 paramètres caractérisant l'anisotropie de l'alliage. Pour normaliser le critère de plasticité par rapport à la direction de laminage, une constante $Q$ doit être définie :

$$
\begin{aligned}
Q^{a} & =\sum_{i=1}^{3}\left(\left|W_{i}\right|-k W_{i}\right)^{a}, \\
W_{i} & =\frac{2}{3} L_{i 1}-\frac{1}{3} L_{i 2}-\frac{1}{3} L_{i 3}, \quad i=1,2,3
\end{aligned}
$$

La procédure d'identification des 9 paramètres du matériau ( $a$ étant fixé à 2 et $L_{11}$ à 1 ) nécessite la réalisation de neuf tests expérimentaux : deux tests de compression à $0^{\circ}$ et $90^{\circ}$ par rapport à la direction de laminage, deux tests de traction à $0^{\circ}$ et $90^{\circ}$, un traction équibiaxiale, un compression équibiaxiale et trois tests de cisaillement pur. En cas d'impossibilité de réaliser tous ces essais, une stratégie d'identification doit être mise en œuvre. Le problème mécanique étant modélisé, la cinématique d'une formulation anisotrope, utilisable quelles que soient les lois de comportement mécanique, doit être définie.

\section{La description cinématique du modèle}

Le suivi de la matière, et donc des axes d'anisotropie au cours de la transformation, permet de modéliser le comportement mécanique des matériaux anisotropes. Les hypothèses suivantes sont émises : ce modèle est 

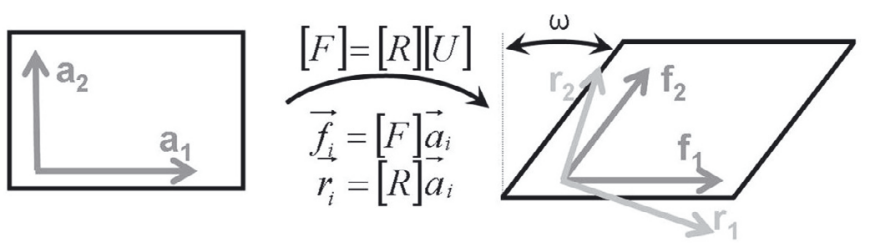

Fig. 1. Évolution des axes d'anisotropie au cours d'un cisaillement simple : réactualisation par $[\mathbf{F}]$ ou par $[\mathbf{R}]$.
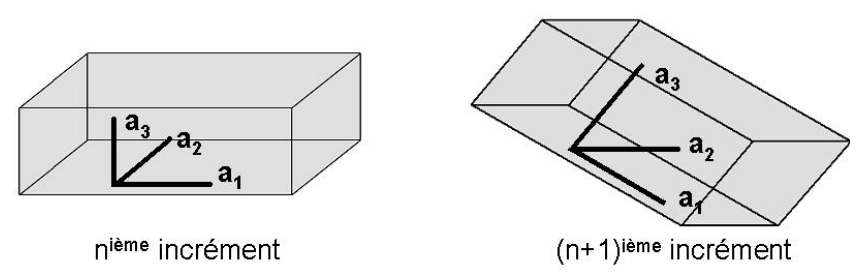

Fig. 2. Évolution des axes d'anisotropie durant la simulation E.F.

développé pour le formage de produits plats avec l'axe 3 d'anisotropie qui reste toujours normal à la tôle et on suppose que les lois de comportement sont exprimées dans la base matérielle $\left\{\boldsymbol{a}_{i}\right\}, i=1,2,3$. Les axes d'anisotropie doivent être réactualisés au cours de la transformation. Pour cela, l'évolution de la matière au cours d'un procédé de formage peut être représentée par le gradient de la transformation $[\mathbf{F}]$, ou par la matrice de rotation rigide $[\mathbf{R}]$, issue de la décomposition polaire $[\mathbf{F}]=[\mathbf{R}][\mathbf{U}],[\mathbf{U}]$ étant le tenseur des déformations pur droit. La comparaison de l'évolution analytique des axes d'anisotropie $\left\{\boldsymbol{a}_{1}\right\}$ et $\left\{\boldsymbol{a}_{2}\right\}$ au cours d'un test de cisaillement simple (Fig. 1) montre que la solution la plus conforme à la réalité est la réactualisation par le gradient de la transformation $[\mathrm{F}]$ (axes $\boldsymbol{f}_{1}$ et $\boldsymbol{f}_{2}$ ).

Les axes d'anisotropie sont donc réactualisés par les relations suivantes au cours de la transformation (Fig. 2) :

$$
\begin{aligned}
\left\{\boldsymbol{a}_{i}^{n+1}\right\} & =\left\|[\boldsymbol{F}]^{n}\left\{\boldsymbol{a}_{i}^{n}\right\}\right\| \quad i=1,2 \\
\left\{\boldsymbol{a}_{3}^{n+1}\right\} & =\left\|\left\{\boldsymbol{a}_{1}^{n+1}\right\} \wedge\left\{\boldsymbol{a}_{2}^{n+1}\right\}\right\|
\end{aligned}
$$

avec $[\boldsymbol{F}]^{n}$ le gradient de la transformation pour l'incrément $n$. Les axes d'anisotropie situés dans le plan de la tôle $\left(\left\{\boldsymbol{a}_{1}\right\},\left\{\boldsymbol{a}_{2}\right\}\right)$ suivent la matière et le dernier axe $\left\{\boldsymbol{a}_{3}\right\}$ est normal aux deux autres, donc au plan de la tôle.

Les équations d'équilibre du problème sont résolues dans le repère de référence $\left(\left\{\boldsymbol{e}_{i}\right\}, i=x, y, z\right)$, alors que les contraintes doivent être calculées dans la base matérielle $\left\{\boldsymbol{a}_{i}\right\}, i=1,2,3$, afin de garder l'objectivité du modèle nécessaire en grandes déformations. En choisissant une réactualisation des axes d'anisotropie par $[\mathbf{F}]$, le repère matériel n'est plus orthogonal. Notre choix d'exprimer les contraintes dans un tel repère découle des travaux récents en structures composites [8] ou d'autres plus anciens sur le formage des structures fines en grandes transformations [9]. Un transport entre la base de référence orthonormée $\left(\left\{\boldsymbol{e}_{i}\right\}, i=x, y, z\right)$ et la base matérielle $\left(\left\{\boldsymbol{a}_{i}\right\}, i=\right.$ $1,2,3)$, supposée covariante dans un espace vectoriel euclidien, doit être défini (Fig. 3). Il faut de plus être vigilant concernant la variances des tenseurs. Pour ce faire

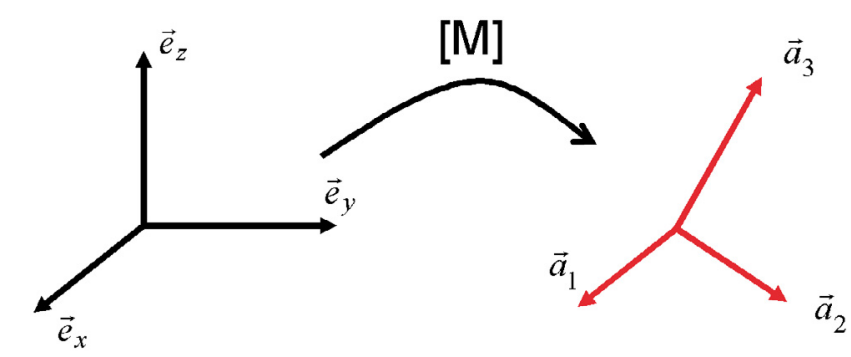

Base référence

Base matériau

Fig. 3. Transport des grandeurs tensorielles entre la base globale $\left(\left\{\boldsymbol{e}_{i}\right\}, i=x, y, z\right)$ et la base matérielle $\left(\left\{\boldsymbol{a}_{i}\right\}, i=1,2,3\right)$.

on définit le déviateur des contraintes $[\boldsymbol{S}]$ comme contravariant et le déviateur des déformations $[\boldsymbol{\epsilon}]$ comme covariant. Les relations de transport [10] de ces grandeurs entre les deux bases du modèle sont dépendantes de la variance des tenseurs et du sens du changement de base (de la base de référence à la base matérielle, ou inversement). On définit donc une matrice de changement de base $[\mathbf{M}]$ permettant de travailler dans la base locale à partir des vecteurs directeurs des axes d'anisotropie $\left\{\boldsymbol{a}_{i}\right\}$.

$$
[\mathbf{M}]=\left[\begin{array}{lll}
a_{1}(1) & a_{2}(1) & a_{3}(1) \\
a_{1}(2) & a_{2}(2) & a_{3}(2) \\
a_{1}(3) & a_{2}(3) & a_{3}(3)
\end{array}\right]
$$

\section{Implémentation du critère de Cazacu}

Ce modèle a été implémenté dans le logiciel E.F. Forge ${ }^{\circledR}$. Toutes les grandeurs sont exprimées dans la base matérielle, comme le souligne l'indice « mat ». La matrice de changement de base $[\mathbf{M}]$ permet le transport entre la base de référence et la base matérielle. Les indices « $n$ » et $« n+1 »$ indiquent le début et la fin de l'incrément. Un algorithme du retour radial [11] permet de calculer l'incrément de contrainte. Au début de l'incrément, le tenseur des taux de déformation $\{\dot{\boldsymbol{\epsilon}}\}^{n+1}$ et le tenseur des contraintes à l'incrément précédent $\{\boldsymbol{S}\}^{n}$ sont disponibles. Les axes d'anisotropie sont réactualisés par le gradient de la transformation. L'étape de prédiction élastique suppose que les déformations au cours de l'incrément sont uniquement élastiques. L'incrément de contrainte $\left\{\dot{\boldsymbol{S}}_{\text {elas }}^{n+1}\right\}$ est donc calculé :

$$
\left\{\dot{\boldsymbol{S}}_{\text {elas }}\right\}^{n+1}=[\boldsymbol{C}]^{-1}\{\dot{\boldsymbol{\epsilon}}\}^{n+1}
$$

Le critère de plasticité $F_{\mathrm{p}}$ est réactualisé. Si $F_{\mathrm{p}} \leq 0$, le comportement est élastique et l'incrément de contrainte déduit du prédicteur élastique $\left(\{\dot{\boldsymbol{S}}\}^{n+1}=\left\{\dot{\boldsymbol{S}}_{\text {elas }}\right\}^{n+1}\right)$. Autrement, la transformation est élasto-plastique et guidée par le système suivant, avec $\dot{\lambda}^{\mathrm{p}}$ le multiplicateur plastique :

$$
\left\{\begin{array}{l}
\left\{\dot{\boldsymbol{\epsilon}}_{\text {mat }}\right\}^{n+1}=[\boldsymbol{C}]^{-1}\left\{\dot{\boldsymbol{S}}_{\text {mat }}\right\}^{n+1}+\dot{\lambda}^{p} \frac{\partial f}{\partial \boldsymbol{S}_{\text {mat }}^{n+1}} \\
F_{\mathrm{p}}=f\left(\boldsymbol{S}_{\text {mat }}-\boldsymbol{X}_{\text {mat }}\right)-\sigma_{y}=0
\end{array}\right.
$$


On ne peut exprimer la normale à la surface de plasticité en fonction des composantes du déviateur des contraintes. Il faut donc appliquer au système (8) un développement limité au premier ordre. La notation « $\Delta A »$ représente la valeur « $\dot{A}$.d $t »$. On obtient donc pour l'équation (1) :

$$
\begin{aligned}
\{\dot{\epsilon}\} & -[\boldsymbol{C}]^{-1}\{\Delta \boldsymbol{S}\}-\left.\Delta \lambda \frac{\partial f}{\partial \boldsymbol{S}}\right|_{n} \\
& -\left([\boldsymbol{C}]^{-1}-\left.\Delta \lambda \frac{\partial^{2} f}{\partial \boldsymbol{S}^{2}}\right|_{n}\right) \mathrm{d} \Delta \boldsymbol{S}-\left.\frac{\partial f}{\partial \boldsymbol{S}}\right|_{n} \mathrm{~d} \Delta \lambda=0
\end{aligned}
$$

De la même manière, on obtient l'expression de l'équation (2):

$$
F_{\mathrm{p}}\left(\left\{\boldsymbol{S}_{n+1}\right\}, \bar{\epsilon}_{n+1}, \dot{\bar{\epsilon}}\right)+\left.\frac{\partial F_{\mathrm{p}}}{\partial \boldsymbol{S}}\right|_{n} \mathrm{~d} \Delta \boldsymbol{S}-\frac{\partial \sigma_{y}}{\partial \Delta \lambda} \mathrm{d} \Delta \lambda=0
$$

La combinaison des deux développements limités permet l'obtention des corrections appliquées sur l'incrément de contrainte $\Delta S$ et sur le multiplicateur plastique $\Delta \lambda$.

$$
\begin{gathered}
\mathrm{d} \Delta \lambda=\frac{F_{\mathrm{p}}+\frac{\partial f}{\partial \boldsymbol{S}}[\boldsymbol{P}]^{-1}\left(\{\Delta \boldsymbol{\epsilon}\}-[\boldsymbol{C}] \Delta \boldsymbol{S}-\Delta \lambda \frac{\partial f}{\partial \boldsymbol{S}}\right)}{\frac{\partial f}{\partial \boldsymbol{S}}[\boldsymbol{P}]^{-1} \frac{\partial f}{\partial \boldsymbol{S}}+\frac{\partial \sigma_{y}}{\partial \Delta \lambda}} \\
\mathrm{d} \Delta \boldsymbol{S}=[\boldsymbol{P}]^{-1}\left(\{\Delta \boldsymbol{\epsilon}\}-[\boldsymbol{C}] \Delta \boldsymbol{S}-(\Delta \lambda+\mathrm{d} \Delta \lambda) \frac{\partial f}{\partial \boldsymbol{S}}\right) \\
{[\boldsymbol{P}]=\left([\boldsymbol{I}]+\Delta \lambda[\boldsymbol{C}] \frac{\partial^{2} f}{\partial \boldsymbol{S}^{2}}\right)^{-1}[\boldsymbol{C}]}
\end{gathered}
$$

où $[\mathbf{I}]$ représente la matrice identité de taille 6 . Les dérivées première et seconde du critère de plasticité doivent être calculées pour $\left\{\boldsymbol{S}^{n}+\Delta \boldsymbol{S}\right\}$. Les corrections successives sur $\Delta \boldsymbol{S}$ et $\Delta \lambda$, associées à un algorithme de recherche linéaire, permettent d'atteindre la convergence [12]. Le solveur de Forge ${ }^{\circledR}$ est implicite, ce qui implique le calcul du module tangent $\left[\boldsymbol{B}_{\text {mat }}\right]$ (Éq. (14)), solution de l'équation différentielle obtenue en dérivant le critère de plasticité $F_{\mathrm{p}}$, puis en la combinant avec l'équation (1) du système (8):

$$
\left[\boldsymbol{B}_{\text {mat }}\right]=\frac{\partial \Delta \boldsymbol{S}}{\partial \Delta \boldsymbol{\epsilon}}=[\boldsymbol{P}]-\frac{[\boldsymbol{P}] \frac{\partial f}{\partial \boldsymbol{S}} \otimes \frac{\partial f}{\partial \boldsymbol{S}}[\boldsymbol{P}]}{\frac{\partial f}{\partial \boldsymbol{S}}[\boldsymbol{P}] \frac{\partial f}{\partial \boldsymbol{S}}-\left(\frac{\partial \sigma_{y}}{\partial \overline{\boldsymbol{\epsilon}}}+\frac{1}{\Delta t} \frac{\partial \sigma_{y}}{\partial \dot{\bar{\epsilon}}}\right)}
$$

Le module tangent est exprimé dans le repère matériel et doit donc être transporté dans le repère de référence grâce à la matrice de changement de base $[\mathbf{M}]$ (Éq. (6)).

\section{Résultats}

L'identification des paramètres matériau permettant la modélisation d'un alliage de titane commercialement pur a été réalisée à partir d'une base de données expérimentales réduites. Ne disposant pas de toutes les valeurs expérimentales permettant l'identification directe du critère de plasticité, seuls les résultats de tests de traction uniaxiale dans cinq directions différentes $\left(0^{\circ}\right.$, $30^{\circ}, 45^{\circ}, 60^{\circ}, 90^{\circ}$ par rapport à la direction de laminage), ont été utilisés pour la modélisation (Fig. 4). Le critère de plasticité de Cazacu est identifié à partir des limites élastiques dans les cinq directions. On minimise la fonction $\phi$ (Éq. (15)) qui exprime la différence au sens des moindres carrés entre la contrainte équivalente dans chaque direction $\left(f\left(\boldsymbol{S}_{i}\right)\right)$ et la contrainte dans la direction de laminage $\sigma_{0}$, prise comme référence :

$$
\phi=\sum_{i=1}^{5} \frac{\left(f\left(\boldsymbol{S}_{i}\right)-\sigma_{0}\right)^{2}}{\sigma_{0}^{2}}
$$

L'écrouissage isotrope $\sigma_{y}$, identifié sur la courbe de traction dans la direction de laminage, est défini grâce à une loi complexe adoucissante [13] :

$$
\sigma_{y}=\sqrt{3} K\left(\epsilon_{0}+\bar{\epsilon}_{\mathrm{p}}\right)^{n} \mathrm{e}^{-r \bar{\epsilon}_{p}}+K_{\mathrm{sat}}\left(1-\mathrm{e}^{-r \bar{\epsilon}_{\mathrm{p}}}\right)
$$

Des simulations de traction ont été réalisées pour vérifier la qualité de la modélisation, notamment de l'identification des paramètres matériau pour le critère de plasticité et l'écrouissage isotrope. On compare donc les forces de traction sur le mors mobile, obtenues numériquement et expérimentalement (Fig. 5). On remarque une bonne corrélation pour la direction de laminage. Pour les autres directions, l'écrouissage ne permet pas d'avoir un bon accord entre la simulation et l'expérience. Pour diminuer ce problème, l'écrouissage isotrope doit être dépendant de la direction de chargement afin de reproduire le durcissement mécanique différent dans chaque direction. Des simulations de gonflage elliptique ont été comparées à des cartographies de répartition de déformation et d'épaisseur, obtenues par corrélation d'image sur des essais réalisés sur l'alliage de titane. La déformation plastique maximale observées expérimentalement est de 0,629 , alors que la prédiction numérique est de 0,618 (Fig. 6). Concernant l'épaisseur finale, les mesures effectués par analyse d'image donnent une réduction d'épaisseur de $67 \%$ (0,81 mm), contre $0,85 \mathrm{~mm}$ pour la simulation numérique. On retrouve donc une bonne corrélation entre les mesures et le modèle numérique. La simulation d'un godet met en évidence l'avantage de l'utilisation d'un critère de plasticité anisotrope de Cazacu 2006. À partir d'un critère identifié sur les contraintes d'écoulement plastique, on obtient une géométrie finale et donc des « cornes » d'emboutissage compatibles avec les coefficients de Lankford obtenus avec les tests de traction uniaxiale (Fig. 7). En effet, une relation entre l'évolution du coefficient de Lankford suivant l'orientation par rapport à la direction de laminage et la position des cornes d'emboutissage permet de verifier la fiabilité d'une simulation numérique. Un profil strictement croissant des coefficients de Lankford entre la direction de laminage et la direction transverse implique l'apparition d'une corne d'emboutissage orienté à $90^{\circ}$. 


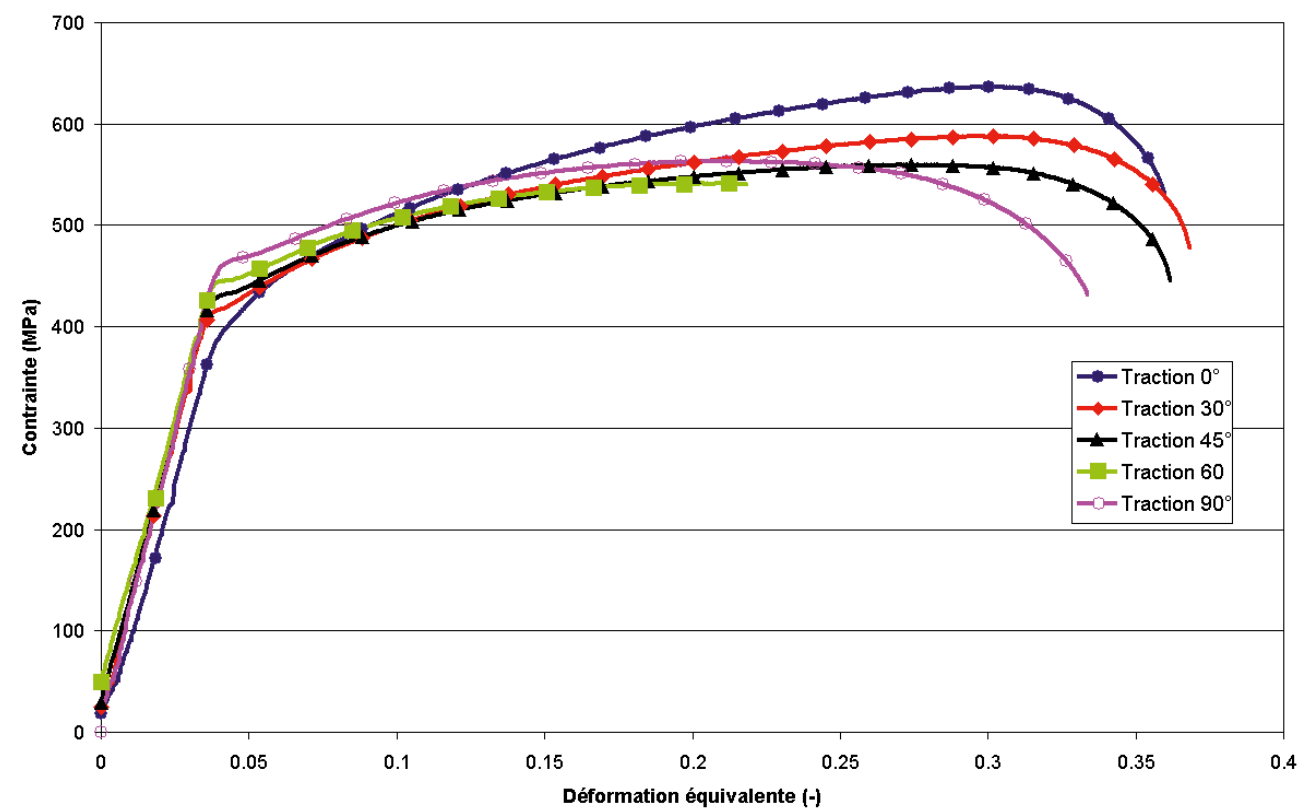

Fig. 4. Courbes contrainte déformation issues d'essais de traction uniaxiale dans cinq directions.

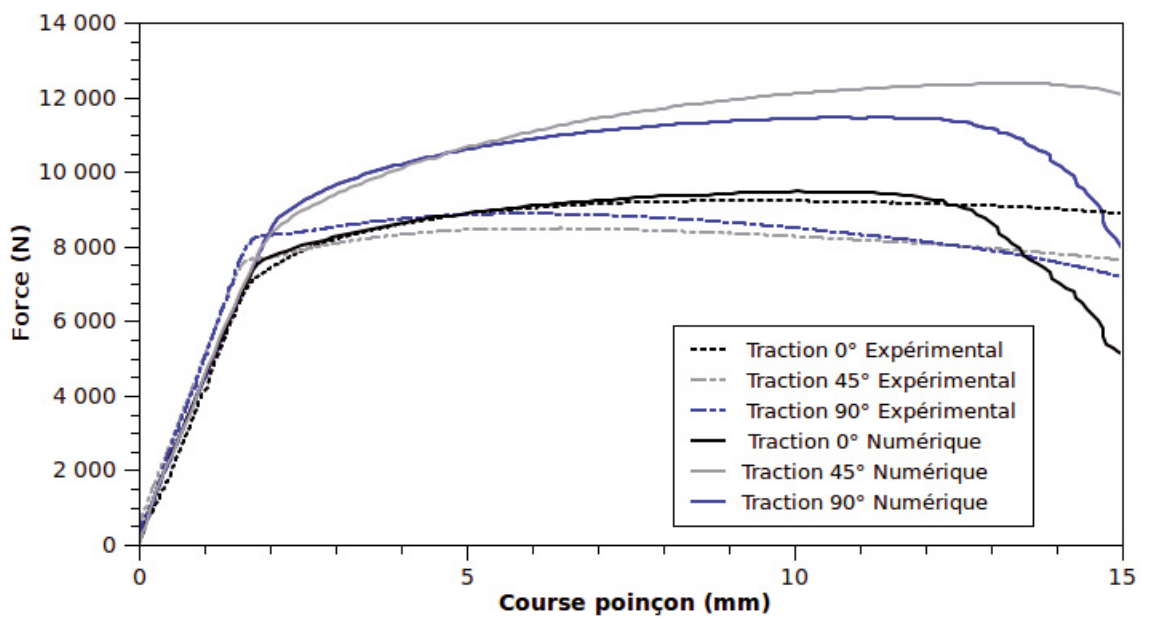

Fig. 5. Test de traction uniaxiale : comparaison entre la simulation numérique et les donnéees expérimentales pour la force de traction.
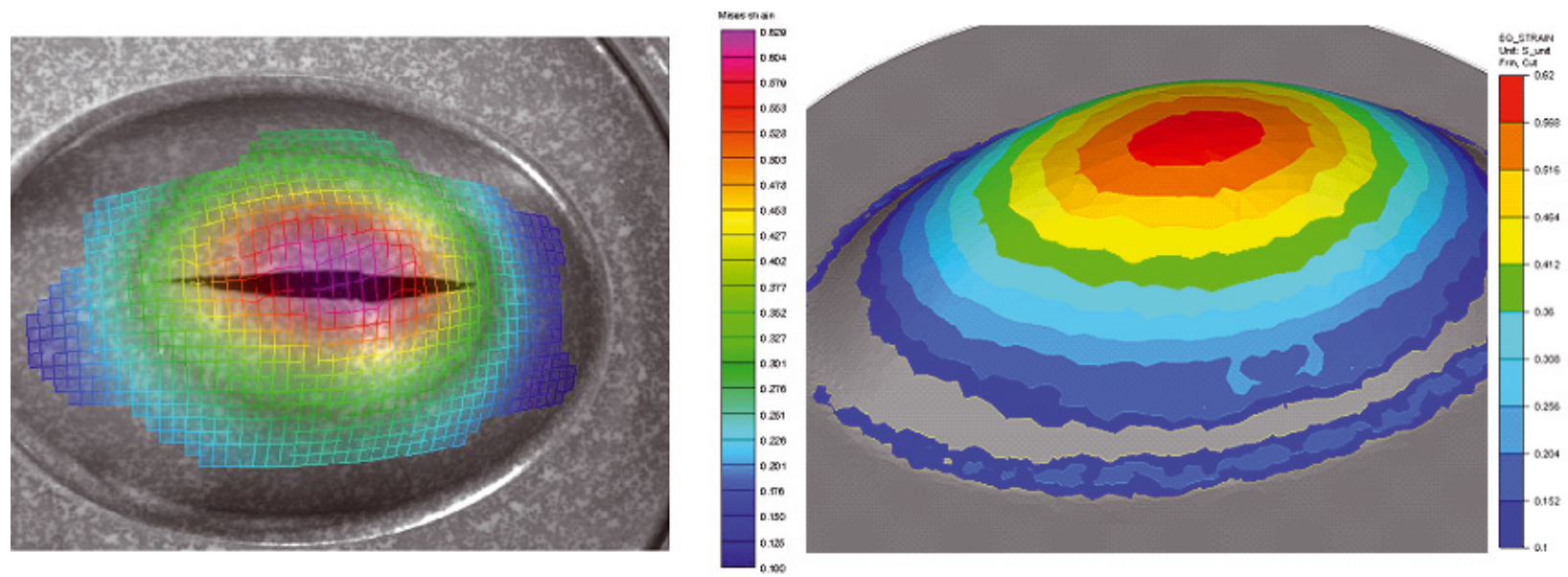

Fig. 6. Comparaison entre la simulation et l'expérimental pour le test de gonflage elliptique : déformation équivalente (échelle semblable pour les deux figures). 


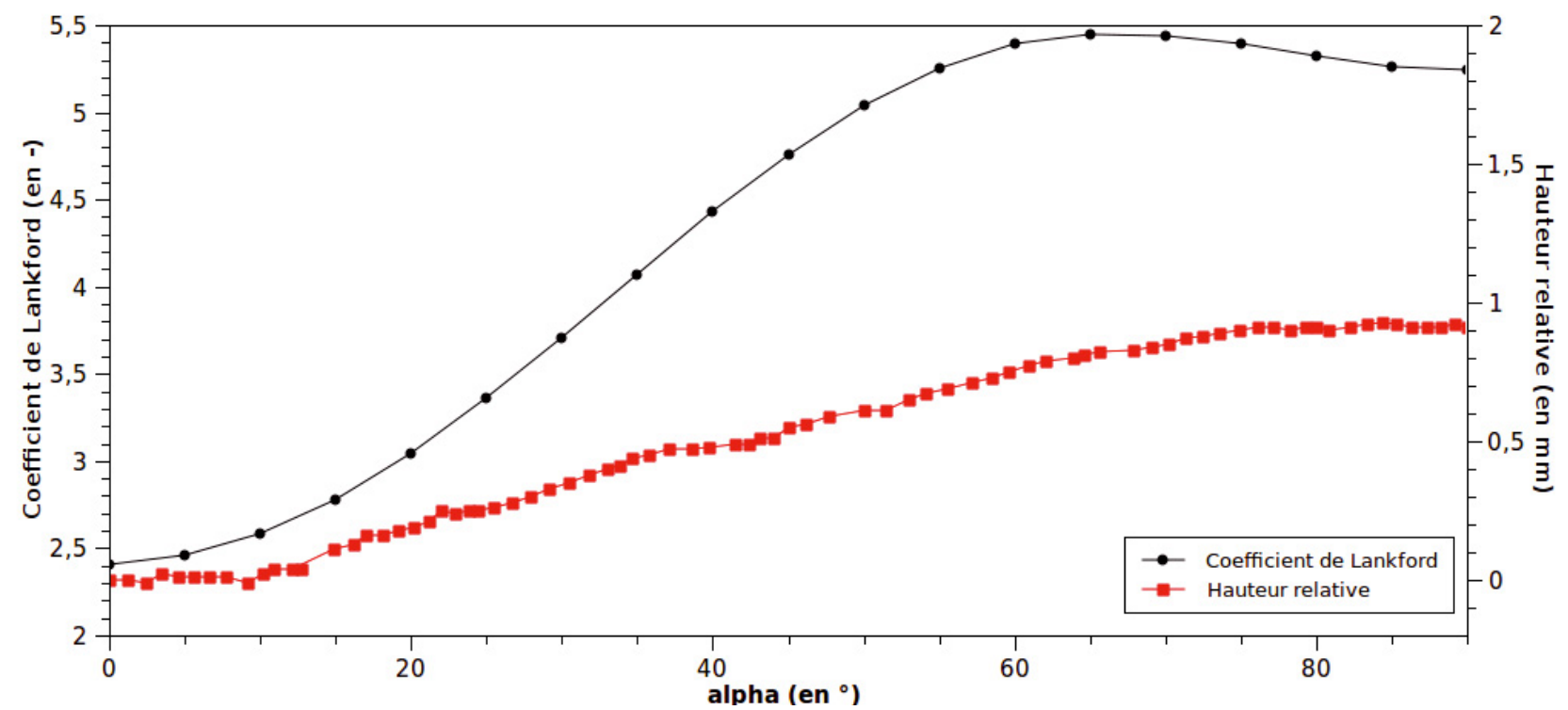

Fig. 7. Courbe de la hauteur du godet adimensionnalisé et des coefficients de Lankford en fonction de l'angle par rapport à la direction de laminage.

\section{Conclusion}

Un nouveau modèle permettant la modélisation et la simulation du comportement anisotrope des tôles a été développé et implémenté dans un logiciel E.F. Forge ${ }^{\circledR}$. Les axes d'anisotropie situés dans le plan de la tôle suivent la transformation de la matière. Le modèle mécanique est défini par un critère de plasticité, une loi d'écrouissage isotrope et un écrouissage cinématique. Le critère de Cazacu 2006, basé sur une fonction non quadratique, a été utilisé pour modéliser le comportement d'un alliage de titane commercialement pur de type $\alpha$ (structure hexagonal compact). L'identification des paramètres du critère de plasticité et de la loi d'écrouissage résulte de tests de traction uniaxiale. Des simulations de traction, gonflage elliptique et emboutissage ont permis de tester le modèle implémenté, une bonne corrélation entre les résultats numériques et expérimentaux a été observée. Le critère de Cazacu permet de modéliser à la fois le comportement en terme de contrainte, mais aussi de prévoir la géométrie finale pour les surfaces libres. Ce point lui procure un net avantage comparé à des critères plus simples tel le critère de Hill 1948, qui est incapable de modéliser à la fois le comportement mécanique et la géométrie finale avec un même jeu de paramètres pour des coefficients de Lankford très différents selon les directions. Cependant l'utilisation d'un écrouissage isotrope dépendant de la direction de sollicitation permettrait de mieux décrire le comportement macroscopique du matériau, notamment la réponse au test de traction.

Remerciements. Ce travail est réalisé dans le cadre du projet OPTIMIST, financé par la Fondation de Recherche pour l'Aéronautique et l'Espace.

\section{Références}

[1] O. Cazacu, B. Plunkett, F. Barlat, Orthotropic yield criterion for hexagonal closed packed metals, Int. J. Plasticity 22 (2006) 1171-1194

[2] G. Lütjering, J.C. Williams, Titanium, Springer, 2nd edition, 2007

[3] R. Hill, Constitutive modelling of orthotropic plasticity in sheet metals, J. Mech. Phys. Solids 38 (1990) 405-417

[4] F. Barlat, J.W. Yoon, O. Cazacu, On the linear transformations of stress tensors for the description of plastic anisotropy, Int. J. Plasticity 23 (2007) 876-896

[5] L. Duchêne, T. Lelotte, P. Flores, S. Bouvier, A.M. Habraken, Rotation of axes for anisotropic metal in fem simulations, Int. J. Plasticity 24 (2008) 397-4427

[6] J.L. Chaboche, A review of some plasticity and viscoplasticity constitutive theories, Int. J. Plasticity 24 (2008) 1642-1693

[7] M. Ben Tahar, E. Massoni, Use of the modified maximum force criterion for necking analysis during sheet bulging, 10th International Metal Forming Conference 2004, p. 229-234

[8] X.Q. Peng, J. Cao, A continuum mechanics based on orthogonal constitutive model for woven composite fabrics, Compos. Part A-Appl. S. 36 (2005) 859-874

[9] K. Mattiasson, On the co-rotational finite element formulation for large deformation problems, Ph.D. Thesis Chalmers University of Technology, 1983

[10] G.A. Holzapfel, Nonlinear Solid Mechanics: A continuum approach for engineering, Wiley, 1st edition, 2000

[11] J.C. Simo, R.L. Taylor, A return mapping algorithm for plane stress elastoplasticity, Int. J. Numer. Meth. Eng. 22 (2005) 649-670

[12] R. Knockaert, Numerical and experimental study of the strain localization during sheet forming operations, Ph.D. Thesis, École des Mines de Paris, 2001

[13] A. Gavrus, Identification automatique des paramètres rhéologiques par analyse inverse, Ph.D. Thesis, École des Mines de Paris, 1996 